\title{
Staphylococcus aureus bacteremia in children: Antibiotic resistance and mortality
}

Oren Gordon ${ }^{\mathrm{a}, \mathrm{b}}$, Matan J. Cohen ${ }^{\mathrm{a}, \mathrm{d}}$, Itai Gross ${ }^{\mathrm{c}}$, Sharon Amita, Dina Averbuch ${ }^{\mathrm{a}, \mathrm{b}}$, Dan Engelhard ${ }^{\mathrm{a}, \mathrm{b}}$ and Allon E. Moses ${ }^{\mathrm{a}}$.

Departments of Clinical Microbiology and Infectious Diseases ${ }^{a}$, Pediatrics ${ }^{b}$ and Pediatric Emergency Medicine ${ }^{d}$ at Hadassah-Hebrew University Medical Center, Jerusalem, Israel. Clalit Health Services Jerusalem, Israeld.

Background: Staphylococcus aureus (SA) is a major cause of bacteremia in children. Methicillin-resistant SA (MRSA) is considered a public health threat; however, difference in prognosis of children suffering of Methicillin-sensitive SA (MSSA) vs. MRSA bacteremia is not well defined.

Methods: Data of all SA bacteremia events in children (0-16 years), from 2002 to 2016, in a single tertiary center were Collected. Positive cultures within 48 hours of hospitalization were considered community

associated (CA). Those obtained afterwards or from children hospitalized within the previous year, were considered Health-care associated (HA).

Results: We recorded 427 events, 284 (66\%) were HA, 64 (15\%) were MRSA and $9(2 \%)$ were CA-MRSA (Table 1). There was no increase in MRSA during the study period (Figure 1). Inhospital, 30-day and one-year mortality were 3\% (12 cases), $3.5 \%$ (16 cases) and 12\% (50 cases) respectively. Prior diagnosis and current site of infection was available for $335(78 \%)$ children (Table 2). Children with HA bacteremia had a much higher

Table 1. Pediatric Staphylococcus aureus bacteremia, 2002-2016, Hadassah medical center, Jerusalem, Israel ( $N=427)$.

\begin{tabular}{|c|c|c|c|c|c|}
\hline & HA-MSSA & HA-MRSA & CA-MSSA & CA-MRSA & p-value \\
\hline Number (\%) & $226(53 \%)$ & $55(12.9 \%)$ & $137(32.1 \%)$ & $9(2.1 \%)$ & \\
\hline Female, n (\%) & $87(38 \%)$ & $22(40 \%)$ & $37(27 \%)$ & $5(55 \%)$ & 0.06 \\
\hline Mean Age, years (SD) & $3.5(4.0)$ & $2.9(3.4)$ & $5.5(4.7)$ & $3.6(4.0)$ & $<0.001$ \\
\hline $\begin{array}{l}\text { Mean duration of hospitalization } \\
\text { before positive culture, days (SD) }\end{array}$ & $10.3(14.4)$ & $20.1(20.3)$ & $0.71(0.49)$ & $0.76(0.48)$ & $<0.001$ \\
\hline $\begin{array}{l}\text { Mean duration of hospitalization } \\
\text { after positive culture, days (SD) }\end{array}$ & $16(17.3)$ & $23(23)$ & $5.74(8.8)$ & $7.2(12.7)$ & $<0.001$ \\
\hline Mortality after $\mathbf{3 0}$ days, $\mathbf{n}(\%)$ & $7(3 \%)$ & $6(11 \%)$ & $3(2 \%)$ & $0(0 \%)$ & \\
\hline In hospital death, $\mathbf{n}(\%)$ & $7(3 \%)$ & $4(7 \%)$ & $1(1 \%)$ & $0(0 \%)$ & \\
\hline
\end{tabular}

Table 2. Prior diagnosis and current site of infection $(\mathbf{N}=335)$

\begin{tabular}{|l|c|c|c|c|c|}
\hline & HA_MSSA & HA_MRSA & CA_MSSA & CA_MRSA & p-value \\
\hline Total & $160(48 \%)$ & $48(14 \%)$ & $119(36 \%)$ & $8(2 \%)$ & \\
\hline Prior chronic disease & $119(74 \%)$ & $38(79 \%)$ & $28(24 \%)$ & $1(13 \%)$ & $<0.001$ \\
\hline $\begin{array}{l}\text { Hemato-oncology and } \\
\text { Immune suppression }\end{array}$ & $60(38 \%)$ & $11(23 \%)$ & $5(4 \%)$ & $0(0 \%)$ & $<0.001$ \\
\hline Congenital malformation & $39(24 \%)$ & $22(46 \%)$ & $16(13 \%)$ & $1(13 \%)$ & $<0.001$ \\
\hline Any concurrent infection & $58(36 \%)$ & $15(31 \%)$ & $81(68 \%)$ & $2(25 \%)$ & $<0.001$ \\
\hline Osteomyelitis and Arthritis & $18(11 \%)$ & $1(2 \%)$ & $46(39 \%)$ & $1(13 \%)$ & $<0.001$ \\
\hline Endocarditis & $5(3 \%)$ & $0(0 \%)$ & $3(3 \%)$ & $0(0 \%)$ & 0.62 \\
\hline Skin and soft tissue infection & $9(6 \%)$ & $3(6 \%)$ & $13(11 \%)$ & $0(0 \%)$ & 0.3 \\
\hline
\end{tabular}

MSSA- Methicillin-sensitive Staphylococcus aureus, MRSA- Methicillin-resistant Staphylococcus aureus, CACommunity associated, HA-Health care associated. See Methods for definitions. rate of prior diagnosis of chronic disease. The Kaplan-Meier survival curves in the first year after positive blood culture is shown in Figure 2A. Comparing 47 pairs of HA-MSSA and HA-MRSA bacteremia events, matched according to length of stay prior bacteremia, showed similar survival rate (Figure 2B). On multivariate analysis controlling for demographics, admitting department and prior morbidity, HA bacteremia and prior chronic disease were associated with increased one-year mortality: $\mathrm{HR}(95 \% \mathrm{Cl})$ $4.1(1.3-12)$ and 3.4 (1.2 to 9.0), respectively. MRSA was not significantly associated with increase one-year mortality compared to MSSA: HR $(95 \% \mathrm{Cl}) 1.4$ (0.6 to 3.1$)$.

Figure 1. Pediatric Staphylococcus aureus bacteremia, 2002-2016

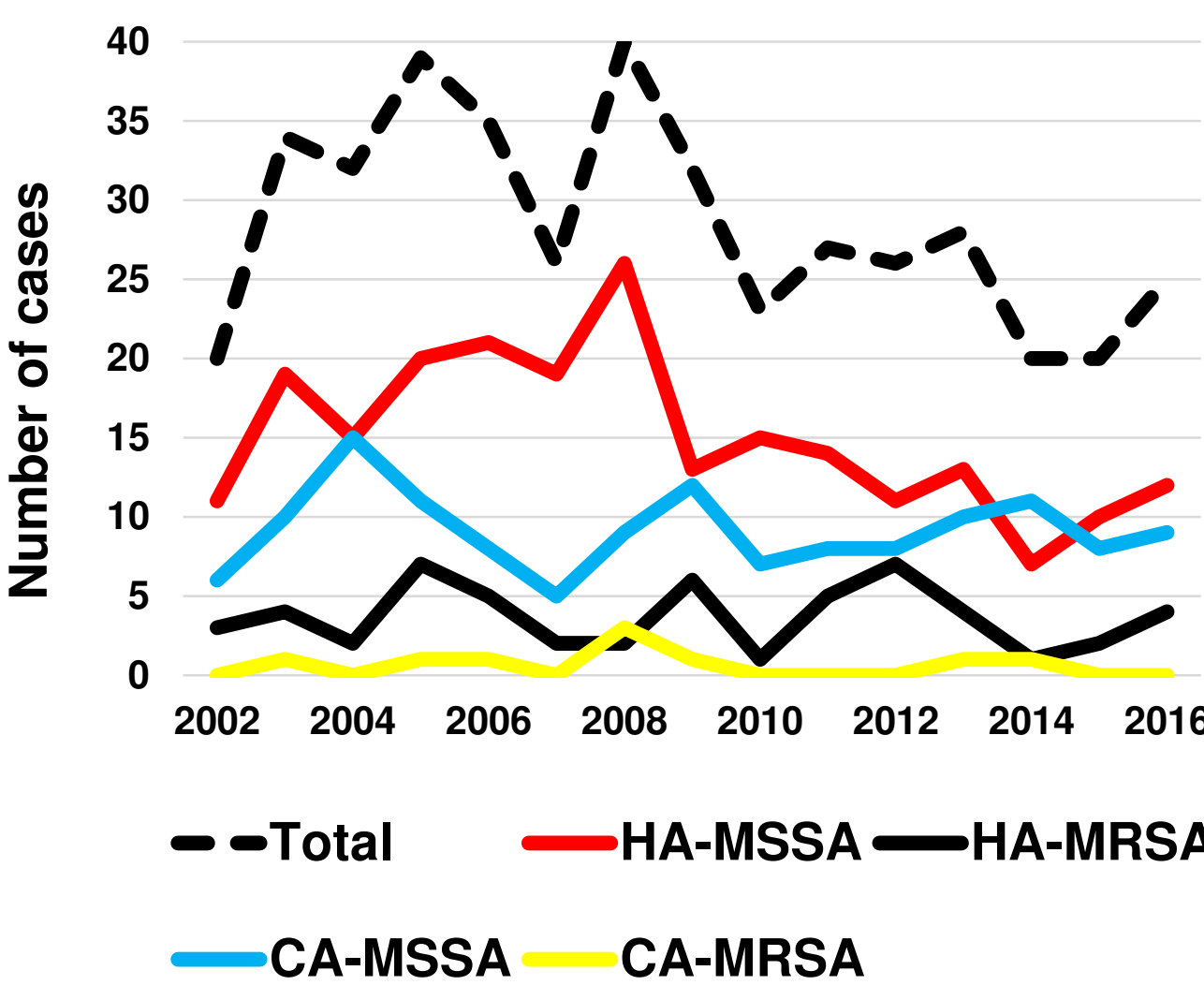

Figure 2. Survival following pediatric Staphylococcus aureus bacteremia
A

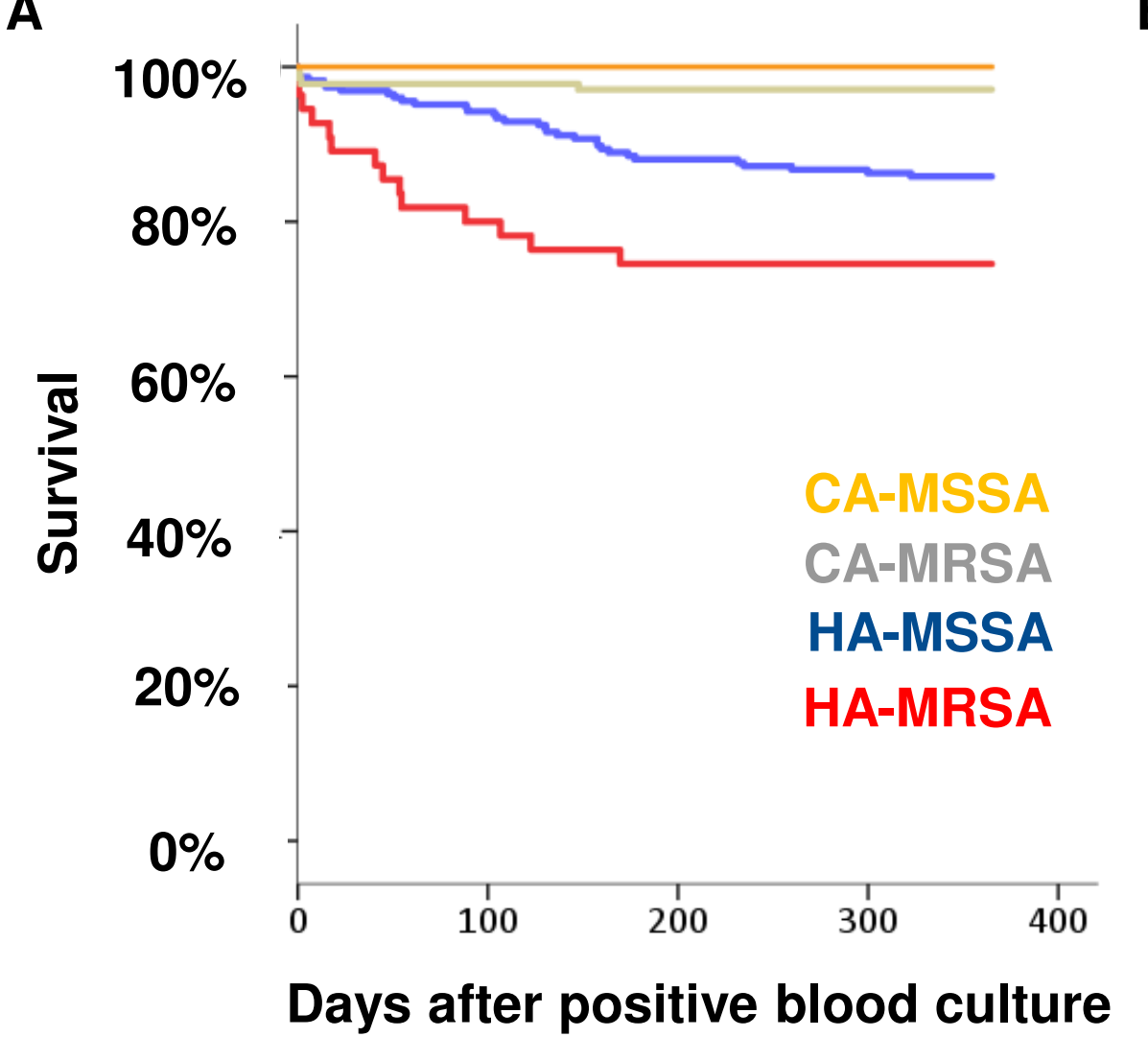

B

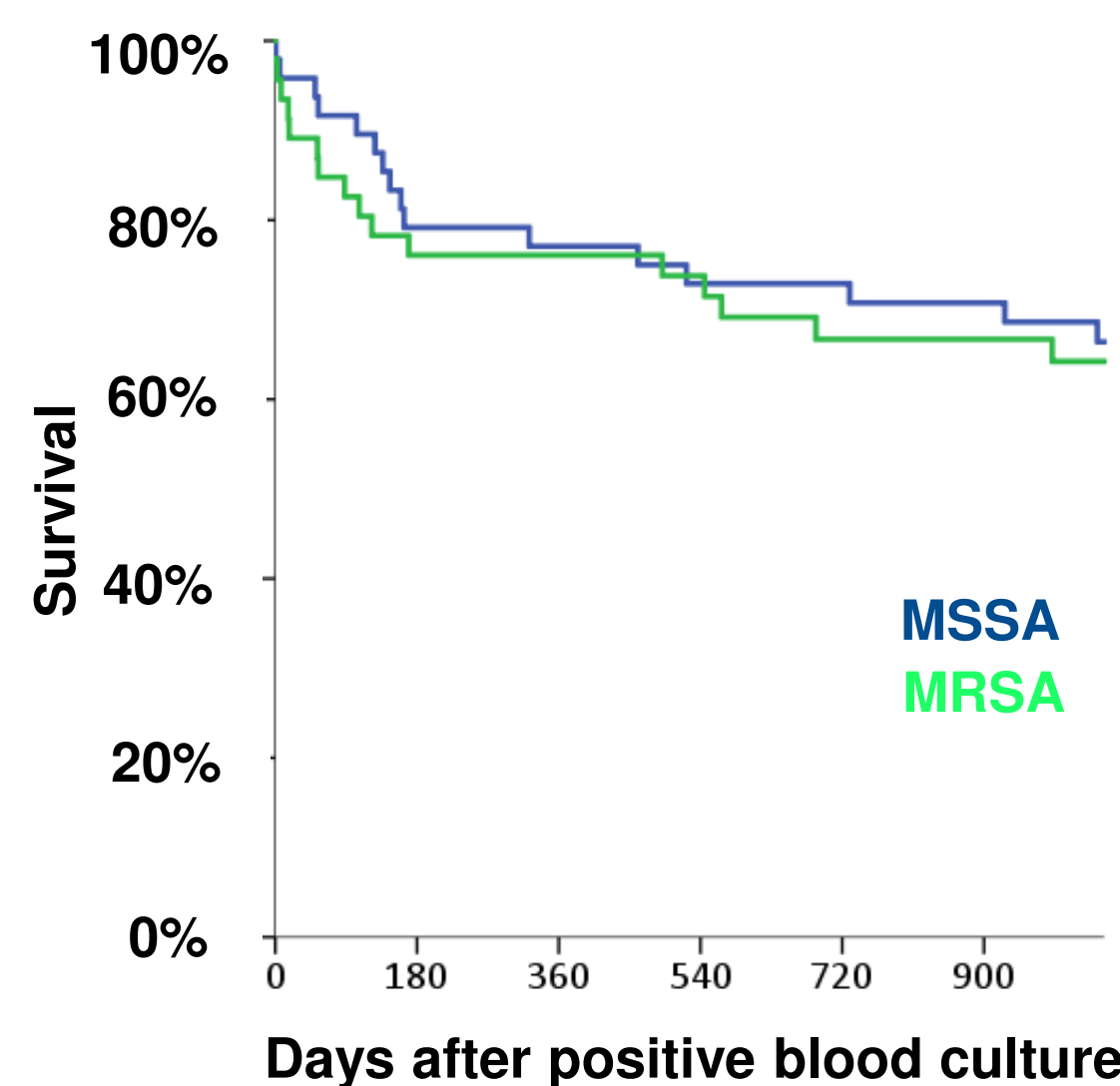

Conclusions: Short term pediatric mortality after SA bacteremia is low. HA-SA bacteremia bares an increased long-term risk for mortality, particularly in children suffering chronic disease. Mortality was not increased for MRSA compared to MSSA bacteremia. The very low rate of CA-MRSA bacteremia justifies our current practice not to include Glycopeptides in the empiric treatment of CA bacteremia. 